Pacific Journal of Mathematics

AN ABSTRACT POTENTIAL THEORY WITH CONTINUOUS 


\title{
AN ABSTRACT POTENTIAL THEORY WITH CONTINUOUS KERNEL
}

\author{
H. S. BEAR
}

1. Introduction. In the study of complex function algebras, it is a standard technique to consider the functions as being defined on the spectrum (maximal ideal space) of the algebra. In other words, one routinely replaces a function algebra $A$ by its Gelfand representation $\hat{A}$. Recall that the Gelfand representation of a Banach algebra is just the standard representation of any normed space $A$ as a family of functionals on $A^{*}$. Each $x \in A$ is represented as the functional $\bar{x}$ on points $F \in A^{*}$ defined by $\bar{x}(F)=F(x)$. The Gelfand representation simply restricts the domain of $\bar{x}$ to the very small set consisting of those $F \in A^{*}$ which are multiplicative (i.e., to the homomorphisms of the algebra $A$ ). Of course this restriction is necessary if $\hat{A}$ is to be again an algebra. However, a fair amount of structure accrues to the representation by virtue of this restriction (cf. [17]).

To consider the standard example, let $A$ be the algebra of continuous complex valued functions on the unit circle in the complex plane which have analytic extensions to the unit disc. Then the spectrum $S_{A}$ is (homeomorphic to) the disc, and the representation $\bar{f}$ gives the analytic extension for each $f \in A$. Now consider the space $C$ of all continuous real functions on the unit circle. These functions also have natural extensions, as harmonic functions, to the unit disc. It follows that the disc is embedded as a compact subset $\Sigma$ of $C^{*}$, and that the harmonic extensions appear as functionals on $C^{*}$ restricted to this set $\Sigma$. In this setting, the disc is not a unique set to which the functions extend "naturally," since the circle can be put on other Riemann surfaces on which the Dirichlet problem is solvable.

In this paper we present axioms for a subset $\Sigma$ of $C^{*}$, where $C=C(\Gamma)$ for an arbitrary compact space $\Gamma$, so that the representation described above does give an effective generalization of the classical potential theory on the disc or sphere in $n$-space. The theory we develop in this way is quite different in intent from those developed in recent years by Bauer, Brelot, and others (cf. [1], [2], [7]). In particular, we start with assumptions which insure that a global Dirichlet problem is automatically solvable.

Our set $\Sigma$ in $C^{*}$ consists of positive continuous functions $z$ on $\Gamma$ weighting a given positive measure $\mu$ on $\Gamma$. That is, we restrict the canonical representation of $C$ as functionals on $C^{*}$ to a subspace of

Received May 31, 1963. This research was supported in part by the National Science Foundation, under grant NSF-GP-216. 
$C^{*}$ consisting of functionals of the form $z \mu$, where for each $u \in C$ we have $(z \mu)(u)=\int u z d \mu$. The functionals $z \mu$ are generalizations of the Poisson measures on the circle or sphere, and the representation $\bar{u}$ on $\Sigma$ obtained for each $u \in C$ is a generalized solution of the Dirichlet problem with boundary value $u$. A surprising amount of the classical theory of harmonic functions on the disc or sphere turns out to depend on the purely topological assumptions we make.

2. Basic assumptions. We list here a set of assumptions and some notation which will be used throughout.

Let $\Gamma$ be a compact Hausdorff space with topology $\mathscr{T}$.

Let $C=C(\Gamma)$ be the linear space of all continuous real-valued functions on $\Gamma$, with the topology $\mathscr{T}_{u}$ of uniform convergence. The uniform norm in $C$ is denoted $\|u\|$.

Assume there is given a positive probability measure $\mu$ on the Baire sets of $\Gamma$. In addition, we are given a set $\Delta$ of strictly positive continuous functions $z$ on $\Gamma$ such that

$$
\int z(\theta) d \mu(\theta)=1
$$

for all $z \in \Delta$. The function identically one is assumed to be in $\Delta$, and is denoted $z_{0}: z_{0}(\theta) \equiv 1$. Hence the measures $z \mu$, for $z \in \Delta$, are functionals of norm one in $C^{*}$, and include $\mu=z_{0} \mu$.

We want to extend the functions $u \in C$ to a compact set containing $\Gamma$, and consisting of $\Gamma$ and the points represented by the continuous kernels $z \in \Delta$. We do this by representing $C$ as a space of continuous functions on a subset of $C^{*}$ consisting of evaluation functionals, and the functionals $z \mu$. Accordingly, define $\Gamma^{*}=\left\{e_{\theta}: \theta \in \Gamma\right\}$, where $e_{\theta}(u)=u(\theta)$ for all $u \in C$. Similarly, let $\Delta^{*}=\{z \mu: z \in \Delta\}$, where $z \mu(u)=\int u z d \mu$ for all $u \in C$. We let $\Sigma^{*}=\Gamma^{*} \cup \Delta^{*}$, and introduce the axioms below on $\Sigma^{*}, \Delta^{*}, \Gamma^{*}$ and $\mu$. Unless otherwise specified, the topology in $C^{*}$, and subsets thereof, is the $w^{*}$ topology, $\mathscr{T}^{*}$.

Axiom 1. $\Sigma^{*}=\Delta^{*} \cup \Gamma^{*}$ is a compact set in $C^{*}$.

Axiom 2. $\Gamma^{*}$ is the boundary of $4^{*}$ in $\Sigma^{*}$.

Axiom 3. The mapping $z \rightarrow z \mu$ is a homeomorphism of $\Delta, \mathscr{T}_{u}$ onto $\Delta^{*}, \mathscr{T}^{*}$.

The representation of $C$ as functions on $\Sigma^{*}$ is as follows: for each $u \in C$, we define $\bar{u}$ on $\Sigma^{*}$ by

$$
\bar{u}(\zeta)=\zeta(u) \quad\left(\zeta \in \Sigma^{*}\right) .
$$

That is, $\bar{u}\left(e_{\theta}\right)=u(\theta)$ for $\theta \in \Gamma$, and $\bar{u}(z \mu)=\int u z d \mu$ for $z \in \Delta$. For simplicity we will denote the points of $\Delta^{*}$ as $z$ rather than $z \mu$, and write 


$$
\bar{u}(z)=\int u(\theta) z(\theta) d \mu(\theta)
$$

We let $H$ denote the space of all functions $\bar{u}$ on $\Sigma^{*}$, for $u \in C$.

Axiom 1 merely expresses the fact that we want a compact extension of our given space $\Gamma$ (or its homeomorphic image $\Gamma^{*}$ ). The second axiom makes it clear that the Silov boundary of our linear space $H$ is in fact a bona fide topological boundary (cf. [5, p. 229], [2]). Although Axiom 3 appears to be quite strong, it turns out to be exactly the necessary assumption for a theory with jointly continuous kernel. Notice that the axioms above are satisfied in the classical case which we shall consider our model: $\Gamma$ is the unit circle in the plane, $\mu$ is the normalized Lebesgue measure, and $\Delta$ is the set of Poisson kernels.

Lemma 1. $\Sigma^{*}$ is Hausdorff. $\Gamma, \mathscr{T}$ is homeomorphic to $\Gamma^{*}, \mathscr{T}^{*}$. Each $\bar{u} \in H$ is continuous on $\Sigma^{*}$.

Proof. The subspace $\Sigma^{*}$ is Hausdorff since $C^{*}$ is. If $\theta_{n} \rightarrow \theta$ in $\mathscr{T}$, then certainly $u\left(\theta_{n}\right) \rightarrow u(\theta)$ for all $u \in C$, or $e_{\theta_{n}} \rightarrow e_{\theta}$ in $\mathscr{T}^{*}$. The mapping $\theta \rightarrow e_{\partial}$ is therefore a continuous one-to-one mapping on a compact space to a Hausdorff space, and hence a homeomorphism. The $w^{*}$ topology on $C^{*}$ is by definition the weakest such that the functions $\bar{u}$ of (2) are continuous. Therefore the functions $\bar{u}$ are in particular continuous on the subset $\Sigma^{*}$.

LeMma 2. $H$ is a uniformly closed linear subspace of $C\left(\Sigma^{*}\right)$ and $H$ contains the constant functions.

Proof. The functionals of $\Sigma^{*}$ are all of norm one, by (1), and the restriction $H \mid \Gamma^{*}$ can be identified with $C$ on $\Gamma$. Hence uniform convergence on $\Gamma^{*}$ is equivalent to uniform convergence on all of $\Sigma^{*}$, and $H$ is in fact isomorphic and isometric with $C$. The constant functions are in $H$ since $\bar{c}(z)=c$ for all $z \in \Delta$, by assumption (1).

Our axioms are given in terms of $\Gamma^{*}$ and $A^{*}$ as subsets of $C^{*}$ to facilitate the description of a topology on the union $\Gamma^{*} \cup \Delta^{*}$. However, the embedding $\Gamma \cup \Delta \rightarrow \Gamma^{*} \cup \Delta^{*}$ is one-to-one, as we shall show in Lemma 5 . It follows that we can consider our assumptions as statements about a given compact set $\Gamma$ and a distinguished subset $\Delta$ of $C(\Gamma)$. Accordingly, we will drop the stars from $\Gamma^{*}$ and $\Delta^{*}$, and regard $\Sigma=\Gamma \cup A$ as the object under consideration. The points of $\Sigma$ are the points $\theta$ of $\Gamma$, and the points (functions) $z$ of $\Delta$. The topology $\mathscr{T}^{*}$ on $\Sigma$ coincides on $\Gamma$ with the given compact topology $\mathscr{T}$, and on $\Delta$ with the uniform topology $\mathscr{T}_{u}$ of $C$ relativized to $\Delta$. We write $\bar{u}(\theta)=u(\theta)$ for $\theta \in \Gamma$, and $\bar{u}(z)=\int u(\theta) z(\theta) d \mu(\theta)$ for $z \in \Delta$. 
LEMma 3. If $\bar{u}(z)=0$ for all $z \in \Delta$, then $u \equiv 0$. If $\bar{u}(z) \geqq 0$ for all $z \in \Delta$, then $u \geqq 0$.

Proof. Both of these statements are immediate from the facts that $\Delta$ is dense in $\Sigma$ (Axiom 2), and the functions $\bar{u}$ are continuous on $\Sigma$.

Lemma 4. If $U$ is a nonempty open set in $\Gamma$, then $\mu(U)>0$.

Proof. Assume that $\mu(U)=0$ for some nonempty open set $U \subset \Gamma$. Let $u$ be a function in $C$ such that $u=0$ outside $U$, and $u \neq 0$. Then for every $z \in \Delta, \bar{u}(z)=\int u z d \mu=0$, since $u=0$ off $U$, and $\mu=0$ on $U$. This contradicts Lemma 3, and proves the statement.

Lemma 5. The mapping $\Gamma \cup \Delta \rightarrow \Gamma^{*} \cup \Delta^{*}=\Sigma^{*}$ is one-to-one.

Proof. The representation of a functional in $C^{*}$ as a measure on $\Gamma$ is of course unique. The lemma asserts that the representation of this measure in the form $z \mu$ for continuous positive $z$, or the form $e_{\theta}$ (unit point mass at $\theta$ ), is unique. This is clearly the case if (and only if) the support of $\mu$ is all of $\Gamma$.

Since $\Sigma=\Gamma \cup \Delta$ consists of distinct functionals in $C^{*}, H$ is a separating linear subspace of $C(\Sigma)$. Such a subspace has a Silov boundary in $\Sigma$; i.e., a unique minimal closed set $Y$ in $\Sigma$ such that each $\bar{u} \in H$ attains its maximum on Y. ([2], or for an elementary proof, [4]). Since each functional $\zeta \in \Sigma$ has norm one, it is clear that each $\bar{u} \in H$ attains its maximum on $\Gamma$. Moreover, $H \mid \Gamma=C(\Gamma)$, so $\Gamma$ is a minimal closed set with this property. We have proved the following:

THEOREM 1. The Silov boundary for $H$ in $\Sigma$ is the topological boundary $\Gamma$ of $\Delta$ in $\Sigma$.

It is of course true by definition that a maximum principle holds for the functions in $H$ and the Silov boundary $\Gamma$. The fact that $\mu$ is supported by all of $\Gamma$, which follows from the fact that $\Gamma$ is the topological boundary of $\Delta$, allows us to sharpen the maximum principle to strict inequality. This situation also occurs in some function algebras (cf. [3], [13]).

THEOREM 2. (Strict maximum principle) If $\bar{u}(z)=\|\bar{u}\|$ for some $z \in \Delta$, then $\bar{u}$ is a constant.

Proof. Assume that $\bar{u}(z)=\|\bar{u}\|=\|u\|$, and that $u$ is non-constant 
and hence $\bar{u}$ is non-constant). Let $u(\theta)=\|u\|-v(\theta)$, where $v(\theta) \geqq 0$ and $v$ is not identically zero. Let $v(\theta) \geqq \varepsilon>0$ for all $\theta$ in some open set $U \subset \Gamma$. Then

$$
\begin{aligned}
u(z) & =\int u(\theta) z(\theta) d \mu(\theta) \\
& =\int[\|u\|-v(\theta)] z(\theta) d \mu(\theta) \\
& =\|u\|-\int v(\theta) z(\theta) d \mu(\theta) \\
& \leqq\|u\|-\varepsilon \mu(U) \min z \\
& <\|u\| .
\end{aligned}
$$

This contradicts the assumption that $\bar{u}(z)=\|u\|$. Hence $u$ and $\bar{u}$ are constant.

CoROllary. If $\bar{u} \geqq 0$ on $\Sigma$ and $\bar{u}(z)=0$ for some $z \in \Delta$, then $\bar{u} \equiv 0$.

Proof. If $v=\|u\|-u$, then $\bar{v}=\|u\|-\bar{u}$, and $\bar{v}$ assumes its maximum, $\|u\|$, at the point $z \in \Delta$. Hence $\bar{v}$ is a constant, and $\bar{u} \equiv 0$.

THeOREM 3. $\Delta$ is closed in $C$ if no singleton in $\Gamma$ is open and closed. In particular, $\Delta$ is closed in $C$ if $\Gamma$ is connected.

Proof. Let $\left\{z_{n}\right\}$ be a sequence of distinct functions in $\Delta$ which converges uniformly to $w \in C$. We must show that $w \in A$. The uniform convergence of the $z_{n}$ implies that the functionals $z_{n} \mu$ converge in $\mathscr{T}^{*}$ to $w \mu$. Since $\Sigma$ is compact in the $w^{*}$ topolgy, $w \mu \in \Sigma$. Thus either $w \in \Delta$ and we are done, or $w \mu$ is evaluation at some $\theta_{0} \in \Gamma$, for all $u \in C$. For $w \mu$ to be unit point mass at $\theta_{0}$, we must have $\mu\left\{\theta_{0}\right\}>0$, $w\left(\theta_{0}\right)>0$, and $w=0$ on $\Gamma \sim\left\{\theta_{0}\right\}$. This implies that $\left\{\theta_{0}\right\}$ is open, since $w$ is continuous; $\left\{\theta_{0}\right\}$ is automatically closed since $\Gamma$ is Hausdorff.

The following example, which gives the natural "potential theory" in one dimension, shows that the hypothesis on $\Gamma$ in Theorem 3 is necessary.

ExAmple. Let $\Gamma$ consist of the two points -1 and 1 , with the discrete topology. Let $\mu$ assign mass $1 / 2$ to each point. We denote functions $u$ on $\Gamma$ by pairs, $u=(a, b)$, where $a=u(-1), b=u(1)$. The family $\Delta$ will consist of the functions $z_{x}=(1-x, 1+x)$, for $-1<x<1$. The function $z_{0}$ is identically one, and for each function $z_{n}$ we have

$$
\int z_{x} d \mu=(1-x) \frac{1}{2}+(1+x) \frac{1}{2}=1 .
$$


The family $\Gamma^{*} \cup \Delta^{*}$ is clearly homeomorphic to the compact interval $[-1,1]$, and $\Gamma^{*}$ is the boundary of $\Delta^{*}$. Here $\Delta$ is not closed in $C(\Gamma)$, since the function $(0,2)$ is the uniform limit of functions $(1-x, 1+x)$ as $x \rightarrow 1$. The functions $\bar{u}$ can be represented as follows: if $u=(a, b)$, then

$$
\begin{aligned}
\bar{u}\left(z_{x}\right) & =\int u z_{x} d \mu \\
& =a\left(\frac{1-x}{2}\right)+b\left(\frac{1+x}{2}\right) .
\end{aligned}
$$

Hence the graph of $\bar{u}$ is the line joining $(-1, a)$ and $(1, b)$, and $\bar{u}\left(z_{x}\right)$ is the point on this line above $x$.

3. The harmonic functions on $\Delta$. In this section we extend our class $H$ to a class of functions which are "harmonic" on $\Delta$, without necessarily being continuously extendable to all of $\Sigma$. We show that the kernels $P(z, \theta)=z(\theta)$ are harmonic in $z$ for each fixed $\theta$, and that they are extreme points of certain compact convex sets of harmonic functions. With one additional assumption on 4 , which holds in the classical case, we show that the set of differences of positive harmonic functions is isomorphic and homeomorphic with $C^{*}$.

Lemma 6. If $P(z, \theta)=z(\theta)$ for all $z \in \Delta$, all $\theta \in \Gamma$, then $P$ is: jointly continuous on $\Delta \times \Gamma$ with the product topology.

Proof. The statement of the lemma holds for any family (here 4 ) of continuous functions on a compact space, with the uniform topology: $[14$, p. 224].

In connection with the above lemma, it is worth noting that the uniform topology is the weakest such that $P$ is jointly continuous. Thus Axiom 3 is necessary if we are to develope a theory based on the idea of a jointly continuous kernel.

With the above definition of $P$, the representation (3) for functions $\bar{u} \in H$ can be written in the familiar form

$$
\bar{u}(z)=\int u(\theta) P(z, \theta) d \mu(\theta) .
$$

Definition. Let $\mathscr{U}$ be the topology of uniform convergence on compact subsets of $\Delta$ (the u.c.c. topology, or compact-open topology). Let $\mathscr{H}$ denote the $\mathscr{U}$-closure of $H \mid \Delta$. That is, $\mathscr{H}$ is the set of all u.c.c. limits on $\Delta$ of functions in $H$. The functions in $\mathscr{H}$ will be called harmonic. The set $\mathscr{H}$ forms a locally convex real linear topological space with the topology $\mathscr{U}$, since the basic neighborhoods. 
of zero, $\left\{v: \sup _{K}|v(z)|<\varepsilon\right\}$, are convex.

We interrupt our development here to point out explicitly that the family $\mathscr{H}$ just defined is the set of all harmonic functions in the classical case.

Proposition. If $\Gamma=\{z:|z|=1\}, \Delta$ is the set of Poisson kernels on $\Gamma$ (or the open unit disc $\{z:|z|<1\}$, and $\mu$ is normalized Lebesgue measure on $\Gamma$, then $\mathscr{H}$ is the set of all functions on $\Delta$ which are harmonic in the classical sense.

Proof. The proposition is simply the observation that every harmonic function on the open unit disc is the u.c.c. limit of harmonic functions continuous on the closed disc. To see this, let $v$ be harmonic on $\Delta, v+i w$ be analytic on $\Delta$, and $\left\{p_{n}\right\}$ be a sequence of polynomials in $z$ which converge u.c.c. to $v+i w$ on 4 . Then the continuous harmonic functions $\left\{R e p_{n}\right\}$ converge u.c.c. to $v$.

LEMMA 7. $\Delta$ is locally compact, and each harmonic function is continuous on 4 .

Proof. For each $z \in \Delta$ there are disjoint neighborhoods $U$ and $V$ in $\Sigma$ such that $z \in U$ and $\Gamma \subset V$. Hence $U^{-}$is compact, and each point of $\Delta$ has a compact neighborhood $U^{-} \subset \Delta$. Since a harmonic function is a uniform limit of continuous functions on some (compact) neighborhood of each $z \in \Delta$, each $v \in \mathscr{C}$ is continuous on $\Delta$.

LEMMA 8. If $K$ is a compact subset of 4 , then $K$ is an equicontinuous family of functions on $\Gamma$. The functions in $K$ are uniformly bounded, and uniformly bounded away from zero.

Proof. Since $K \subset \Delta$, the hypothesis is that $K$ is compact in the uniform topology $\mathscr{T}_{u} \cdot K$ is therefore a bounded set in the norm \|\| of $\mathscr{T}_{u}$, which means the functions $z \in K$ are uniformly bounded on $\Gamma$. If the functions in $K$ were not uniformly bounded away from zero, then there would be a limit point $z \in K$, since $K$ is compact, with minimum value zero. This minimum value would be attained on the compact set $\Gamma$, which contradicts the assumption that all $z \in \Delta$ are strictly positive. The set $K$ is equicontinuous since the uniform topology $\mathscr{T}_{u}$ is jointly continuous, and $K$ is compact in $\mathscr{T}_{u}[14, \mathrm{p} .233]$.

Definition. We will let $H^{+}$denote the nonnegative functions in $H$, and $\mathscr{H}^{+}$the closure in $\mathscr{U}$ of $H^{+} \mid \Delta$.

THEOREM 4. (Harnack's inequality-see e.g. [8, p. 153]) If $K$ is 
a compact subset of $\Delta$, then there are positive numbers $m$ and $M$ such that for every $v \in \mathscr{H}^{+}$,

$$
m v\left(z_{0}\right) \leqq v(z) \leqq M v\left(z_{0}\right)
$$

for all $z \in K$.

Proof. Recall that the function $z_{0}$ in (5) in identically one: $z_{0}(\theta) \equiv$ 1. We prove that the inequality (5) holds for every $\bar{u} \in H^{+}$, and then the theorem follows by taking uniform limits on the compact set $K \cup\left\{z_{0}\right\}$.

Assume that $u \geqq 0$ on $\Gamma$, and let $\min z=\min \{z(\theta): \theta \in \Gamma\}$. We have

$$
\begin{aligned}
\min z \bar{u}\left(z_{0}\right) & =\min z \int u \cdot 1 d \mu \\
& \leqq \int u z d \mu \\
& =\bar{u}(z) \\
& \leqq\|z\| \int u \cdot 1 d \mu \\
& =\|z\| \bar{u}\left(z_{0}\right) .
\end{aligned}
$$

If $m$ is a uniform lower bound for the functions $z \in K$, and $M$ is a uniform upper bound, then we have

$$
m \bar{u}\left(z_{0}\right) \leqq \bar{u}(z) \leqq M \bar{u}\left(z_{0}\right)
$$

for all $z \in K$, all $\bar{u} \in H^{+}$.

CoRollaRY. (Harnack's second convergence theorem) If $\left\{\bar{u}_{n}\right\}$ is an increasing sequence of functions in $H^{+}$, and $\left\{\bar{u}_{n}(z)\right\}$ is bounded for any $z \in \Delta$, then $\left\{\bar{u}_{n}\right\}$ converges u.c.c. on $\Delta$.

Proof. Suppose that $\bar{u}_{n}(z) \leqq B$ for all $n$, so that the positive series $\sum\left[\bar{u}_{n}\left(z_{1}\right)-\bar{u}_{n-1}\left(z_{1}\right)\right]$ converges. Let $K$ be any compact set in $\Delta$ and let $m$ and $M$ be the constants of Theorem 4 for the set $K \cup\left\{z_{1}\right\}$. Then from (5) we have

$$
\bar{u}_{n}\left(z_{0}\right)-\bar{u}_{n-1}\left(z_{0}\right) \leqq \frac{1}{m}\left[\bar{u}_{n}\left(z_{1}\right)-\bar{u}_{n-1}\left(z_{1}\right)\right],
$$

and hence for all $z \in K$,

$$
\bar{u}_{n}(z)-\bar{u}_{n-1}(z) \leqq \frac{M}{m}\left[\bar{u}_{n}\left(z_{1}\right)-\bar{u}_{n-1}\left(z_{1}\right)\right] .
$$

Therefore the series $\sum\left[\bar{u}_{n}(z)-\bar{u}_{n-1}(z)\right]$ converges uniformly on $K$. 
Since $K$ is arbitrary, this says that $\left\{\bar{u}_{n}\right\}$ converges u.c.c. on $\Delta$.

Notice that the corollary is stated for $H^{+}$, rather than $\mathscr{H}^{+}$. This is because it is not clear that if $v, w \in \mathscr{H}^{+}$, and $v-w \geqq 0$, that $v-w \in \mathscr{C}^{+}$, as would be required in the above proof $\left(\mathscr{H}^{+}\right.$is defined as the set of limits of $\mathrm{H}^{+}$, and not as the positive functions in $\mathscr{H}$ ).

Now we can prove that the kernel $P(z, \theta)$ is harmonic in $z$ for each fixed $\theta \in \Gamma$, and moreover, that each $P(\cdot, \theta) \in \mathscr{P}$..

Theorem 5. If $\theta_{0} \in \Gamma$, then $P\left(\cdot, \theta_{0}\right) \in \mathscr{Z}^{+}$.

Proof. Let $K$ be a compact subset of $\Delta$, and $\varepsilon>0$. We must find $\bar{u} \in H^{+}$such that

$$
\left|\bar{u}(z)-P\left(z, \theta_{0}\right)\right|<\varepsilon
$$

for all $z \in K$.

Since $K$ is an equicontinuous family, there is a neighborhood $U$ of $\theta_{0}$ in $\Gamma$ such that $\left|z(\theta)-z\left(\theta_{0}\right)\right|<\varepsilon$ for all $z \in K$ and all $\theta \in U$. Let $u$ be a nonnegative continuous function on $\Gamma$ such that $u=0$ off $U$, and $\int u d \mu=1$. For $z \in K$ we have

$$
\begin{aligned}
\left|\bar{u}(z)-P\left(z, \theta_{0}\right)\right| & =\left|\int u(\theta) z(\theta) d \mu(\theta)-z\left(\theta_{0}\right)\right| \\
& =\left|\int u(\theta)\left[z(\theta)-z\left(\theta_{0}\right)\right] d \mu(\theta)\right| \\
& \leqq \sup _{\theta \in \bar{U}}\left|z(\theta)-z\left(\theta_{0}\right)\right| \int u(\theta) d \mu(\theta) \\
& <\varepsilon .
\end{aligned}
$$

Since $K$ and $\varepsilon$ are arbitrary, and $\bar{u} \geqq 0, P\left(\cdot, \theta_{0}\right) \in \mathscr{C}^{+}$.

The next two theorems are extensions to our abstract setting of classical results of Herglotz [11], Bray-Evans [6], Evans [9], and Martin [15, p. 153].

THEOREM 6. A function $v$ on $\Delta$ is in $\mathscr{C}^{+}$if and only if there is a positive Baire measure $\nu$ on $\Gamma$ such that for all $z \in \Delta$,

$$
v(z)=\int P(z, \theta) d \nu(\theta) \text {. }
$$

Proof. Assume first that $v$ is given by (6). The integral in (6) can be approximated at any finite number of points $z \in \Delta$ by a Riemann sum of the form

$$
\sum P\left(z, \theta_{i}\right) \nu\left(E_{i}\right) \text {. }
$$

Any function of the form (7) is in $\mathscr{H}^{+}$by Theorem 5. The set of 
functions of the form (7) is equicontinuous on $\Delta$, since

$$
\begin{aligned}
\mid \Sigma P(z, & \left.\theta_{i}\right) \nu\left(E_{i}\right)-\Sigma P\left(z_{1}, \theta_{i}\right) \nu\left(E_{i}\right) \\
& \leqq \Sigma\left|z\left(\theta_{i}\right)-z_{1}\left(\theta_{i}\right)\right| \nu\left(E_{i}\right) \\
& \leqq\left\|z-z_{1}\right\| \Sigma \nu\left(E_{i}\right) \\
& =\left\|z-z_{1}\right\| \nu(\Gamma) .
\end{aligned}
$$

That is, any function of the form (7) will vary by less than $\varepsilon$ on the sphere of radius $\varepsilon / \nu(\Gamma)$ around $z_{1}$. Therefore pointwise convergence of sums (7) will be uniform on any compact set $K \subset \Delta[14$, p. 232]. Hence $v$ is in the $\mathscr{U}$-closure of the $\mathscr{U}$-closed set $\mathscr{H}^{+}$; i.e., $v \in \mathscr{H}^{+}$.

Now assume that $v \in \mathscr{C}^{+}$, and let $\left\{\bar{u}_{\alpha}\right\}$ be a net of functions in $H^{+}$which converges uniformly on compact sets to $v$ :

$$
\begin{aligned}
v(z) & =\lim \bar{u}_{\alpha}(z) \\
& =\lim \int P(z, \theta) u_{\alpha}(\theta) d \mu(\theta) .
\end{aligned}
$$

The measures $\left\{u_{\alpha} \mu\right\}$ are all in some closed ball of $C^{*}$, since

$$
\left\|u_{\alpha} \mu\right\|=\int u_{\alpha}(\theta) d \mu(\theta)=u_{\alpha}\left(z_{0}\right) \rightarrow v\left(z_{0}\right)
$$

(recall that $z_{0}(\theta) \equiv 1$ ). The closed balls in $C^{*}$ are $\mathscr{T}^{*}$ compact, so there is a subnet of $\left\{u_{\alpha} \mu\right\}$ which converges $w^{*}$ to a positive measure $\nu$. For this subnet, also denoted $\left\{u_{\alpha} \mu\right\}$, and the continuous function $P(z, \cdot)$ on $\Gamma$, we have

$$
\begin{aligned}
v(z) & =\lim \int P(z, \theta) u_{\alpha}(\theta) d \mu(\theta) \\
& =\int P(z, \theta) d \nu(\theta) .
\end{aligned}
$$

COROLLARY. $v \in \mathscr{Y}^{+}-\mathscr{H}^{+}$if and only if $v=\int P d \nu$ for some signed Baire measure $\nu$.

Definition. $H_{M}=\left\{\bar{u} \in H: \int|u| d \mu \leqq M\right\}$. Let $\mathscr{H}_{M}$ be the $\mathscr{U}-$ closure of $H_{M} \mid \Delta$.

The hypothesis $v \in \mathscr{H}_{M}$ is our replacement of the classical Fatou condition for harmonic functions in the disc: $(1 / 2 \pi) \int_{0}^{2 \pi}\left|v\left(r e^{i j}\right)\right| d \theta \leqq M$ for all $r<1$ (Fatou [10] or [16, p. 201]). If $\Gamma \stackrel{0}{=}\{z:|z|=1\}, \Delta$ is the set of Poisson kernels, etc., so the classical situation obtains, then $\mathscr{H}_{M}$ is the set of harmonic functions $v$ such that the functions $v_{r}$ are uniformly bounded by $M$ in the $L_{1}$ norm, where $v_{r}\left(e^{i \beta}\right)=v\left(r e^{i \theta}\right)$ (see 
[12, pp. 33-39]). The families $\mathscr{H}_{M}$ are compact sets of harmonic functions (Theorem 8 below), and this compactness accounts for much of their tractability.

Lemma 9. For each $\theta_{0} \in \Gamma, P\left(\cdot, \theta_{0}\right) \in \mathscr{H}_{1}$.

Proof. In the proof of Theorem 5 (that $P\left(\cdot, \theta_{0}\right) \in \mathscr{H}^{+}$) we found for a given compact $K \subset \Delta$ a function $\bar{u} \in H^{+}$such that $\left|\bar{u}(z)-P\left(z, \theta_{0}\right)\right|<\varepsilon$ for all $z \in K$. This function $\bar{u}$ was in $H_{1}$, since $u \geqq 0$ and $\int u d \mu=1$. Thus $P\left(\cdot, \theta_{0}\right)$ is the u.c.c. limit of functions in $H_{1}$, or $P\left(\cdot, \theta_{0}\right) \in \mathscr{H}_{1}$.

For the following theorem in the classical context, see [18, p. 143] or $[12$, p. 33].

THEOREM 7. A function $v$ is in $\mathscr{H}_{M}$ if and only if there is a signed measure $\nu$ on $\Gamma$, with $\|\nu\| \leqq M$, such that for all $z \in \Delta$,

$$
v(z)=\int P(z, \theta) d \nu(\theta) \text {. }
$$

Proof. Assume that $v \in \mathscr{H}_{M}$, and that $\left\{\bar{u}_{a}\right\}$ is a net of functions in $H_{M}$ which converges uniformly to $v$ on compact subsets:

$$
\begin{aligned}
v(z) & =\lim \bar{u}_{\alpha}(z) \\
& =\lim \int P(z, \theta) u_{\alpha}(\theta) d \mu(\theta) .
\end{aligned}
$$

The measures $\left\{u_{\alpha} \mu\right\}$ are all in the $M$-ball of $C^{*}$, since by hypothesis

$$
\left\|u_{a} \mu\right\|=\int\left|u_{a}\right| d \mu \leqq M
$$

As in the proof of Theorem 6, there is a $w^{*}$ accumulation point $\nu$ of $\left\{u_{\alpha} \mu\right\}$, and $\|\nu\| \leqq M$. If $\left\{u_{\alpha} \mu\right\}$ is a subnet converging $w^{*}$ to $\nu$, then

$$
\begin{aligned}
v(z) & =\lim \int P(z, \theta) u_{\alpha}(\theta) d \mu(\theta) \\
& =\int P(z, \theta) d \nu(\theta)
\end{aligned}
$$

for each $z \in \Delta$.

Now assume that $\|\nu\| \leqq M$ and $v$ is given by (7). We showed in the proof of Theorem 6 that $v$ can be uniformly approximated on any compact $K \subset \Delta$ by a finite sum $\Sigma P\left(\cdot, \theta_{i}\right) \nu\left(E_{i}\right)$, where $\left\{E_{i}\right\}$ is a partition of $\Gamma$. By Lemma 9 , each $P\left(\cdot, \theta_{i}\right)$ occuring in this sum can be uniformly approximated (within $\varepsilon / n\|\nu\|$, if there are $n$ summands) by a function $\bar{u}_{i} \in H_{1} \cap H^{+}$. Hence there is a sum $\bar{w}=\Sigma \nu\left(E_{i}\right) \bar{u}_{i}$ which is uniformly close to $v$ on the given compact set $K$. Clearly $\bar{w} \in H$, 
and we have

$$
\begin{aligned}
\int|w| d \mu & \leqq \sum\left|\nu\left(E_{i}\right)\right| \int \bar{u}_{i} d \mu \\
& =\sum\left|\nu\left(E_{i}\right)\right| \\
& \leqq\|\nu\| \\
& =M .
\end{aligned}
$$

Thus $\bar{w} \in H_{M}$, and $v$ can be uniformly approximated on $K$ by functions in $H_{M}$, so $v \in \mathscr{H}_{M}$.

COROLLARY. $\mathscr{H}^{+}-\mathscr{H}^{+}=\bigcup\left\{\mathscr{H}_{M}: M=1,2, \cdots\right\}$.

Proof. This follows from the corollary of Theorem 6 .

LEMMA 10. $H_{M}$ is equicontinuous on $\Delta$.

Proof. If $z, z_{1} \in \Delta$ and $u \in H_{M}$, then

$$
\begin{aligned}
\left|\bar{u}(z)-\bar{u}\left(z_{1}\right)\right| & =\left|\int u(\theta)\left[z(\theta)-z_{1}(\theta)\right] d \mu(\theta)\right| \\
& \leqq\left\|z-z_{1}\right\| \int|u(\theta)| d \mu(\theta) \\
& \leqq M\left\|z-z_{1}\right\| .
\end{aligned}
$$

THEOREM 8. $\mathscr{H}_{M}$ is compact in the topology $\mathscr{C}$.

Proof. The pointwise closure (and, a fortiori, u.c.c. closure) of an equicontinuous family is equicontinuous, and hence $\mathscr{H}_{M}$ is equicontinuous. By Ascoli's theorem [14, p. 233], the subfamily $\mathscr{H}_{\boldsymbol{s}}$ of $C(\Delta)$ is compact in the topology $\mathscr{U}$ if and only if $\mathscr{H}_{M}$ is closed, $\mathscr{H}_{M}$ is equicontinuous, and $\left\{v(z): v \in \mathscr{H}_{M}\right\}$ is bounded for each $z \in \Delta$. We have only the last condition to check. For each $z \in \Delta,\left\{|v(z)|: v \in \mathscr{H}_{M}\right\}$ is bounded by $M\|z\|$, since for $\bar{u} \in H_{M}^{\prime}$,

$$
|\bar{u}(z)| \leqq \int|u(\theta)| z(\theta) d \mu(\theta) \leqq M\|z\|,
$$

and this estimate carries over to $\mathscr{H}_{M}$ on the compact set $\{z\}$.

CoRollary. $\mathscr{H}^{+}-\mathscr{H}^{+}$is $\sigma$-compact.

Proof. This follows from the corollary of Theorem 7.

In the classical case of the unit ball in Euclidean space, the correspondence between functions in $\mathscr{H}^{+}-\mathscr{H}^{+}$and measures is one-to- 
one. The proof uses the specific form of the Poisson kernels $[18, p$. $143,144]$. The uniqueness of a representing measure $\nu$ is of course equivalent to the non-existence of a nontrivial measure orthogonal to all the functions $z \in \Delta$. Restated, the measure corresponding to a function in $\mathscr{C}^{+}-\mathscr{C}^{+}$is unique if the linear span of $\Delta$ is uniformly dense in $C$. We incorporate this hypothesis in the next theorem to make the statement explicit for the classical case.

THEOREM 9. If the linear span of $\Delta$ is uniformly dense in $C$, then the isomorphism $\nu \rightarrow \int P d \nu$ is a homeomorphism of $C^{*}$ onto $\mathscr{H}^{+}-\mathscr{H}^{+}$.

Proof. As noted above the hypothesis contains the assumption that the mapping is one-to-one. Since this isomorphism maps the compact $M$-ball of $C^{*}$ onto the compact set $\mathscr{K}_{M}$, it is sufficient to show the mapping is continuous in either direction. We will show the mapping $v \rightarrow \nu$ from $\mathscr{H}^{+}-\mathscr{H}^{+}$to $C^{*}$ is continuous. Let $v_{a} \rightarrow v$ in the topology $\mathscr{L}$, and let $\nu_{\alpha}, \nu$ be the corresponding measures. Then $\int z d \nu_{a} \rightarrow \int z d \nu$ for all $z \in \Delta$. Since the linear span of $\Delta$ is uniformly dense in $C$, we have $\int g d \nu_{\alpha} \rightarrow \int g d \nu$ for every continuous $g$, or $\nu_{\alpha} \rightarrow \nu$ in $\mathscr{T}^{*}$.

COROLLARY. The extreme points of $\mathscr{H}_{1}$ are the functions $\pm P(\cdot, \theta)$, for $\theta \in \Gamma$.

Proof. These are the images under the isomorphism above of the unit point masses on $\Gamma$ which are the extreme points of the unit ball of $C^{*}$. (The positive extreme points are the minimal positive harmonic functions of R. S. Martin [15].)

\section{REFERENCES}

1. Heinz Bauer, Axiomatische Behandlung des Dirichletschen Problems für elliptische und parabolische Differentialgleichungen, Math. Annalen, 146 (1962), 1-59.

2. Wilovscher Rand und Dirichletsches Problem, Ann. Inst. Fourier, Grenoble, 11 (1961), 89-136.

3. H. S. Bear, A strong maximum modulus theorem for maximal function algebras, T.A.M.S., 92 (1959), 465-469.

4. - The Silov boundary for a linear space of continuous functions, Amer. Math. Mo., 68 (1961), 483-485.

5. H. F. Bohnenblust and S. Karlin, Geometrical properties of the unit sphere of Banach algebras, Annals of Math., 62 (1955), 217-229.

6. H. E. Bray and G. C. Evans, A class of functions harmonic within the sphere, Amer.

J. Math., 49 (1927), 153-180.

7. M. Brelot, Axiomatique des fonctions harmoniques et surharmoniques dans un espace 
localement compact, Sémenaire de théorie du potentiel, 2 (1957-1958), 40 pp (Inst. H. Poincare, Paris).

8. C. Carathéodory, Theory of functions of a complex variable, vol. 1, New York (1954).

9. G. C. Evans, The logarithmic potential (A. M. S. Colloquium Publications, vol. VI) New York (1927).

10. P. Fatou, Séries trigonométriques et séries de Taylor, Acta Math., 30 (1906).

11. G. Herglotz, Ueber Potenzreihen mit positivem reelem Teil im Einheitskreis, Leipz. Ber., 63 (1911).

12. Kenneth Hoffman, Banach spaces of analytic functions, Englewood Cliffs, N. J. (1962).

13. Kenneth Hoffman and I. M. Singer, Maximal algebras of continuous functions, Acta Math., 103 (1960), 217-241.

14. J. L. Kelley, General topology, New York (1955).

15. R. S. Martin, Minimal positive harmonic functions, T.A.M.S., 49 (1941), 137-172.

16. R. Nevanlinna, Eindeutige analytische funktionen, Berlin (1955).

17. Hugo Rossi, The local maximum modulus principle, Annals of Math., 72 (1960), 1-11.

18. M. Tsuji, Potential theory in modern function theory, Tokyo (1959).

UNiversity of CALIFornia, SANTA Barbara 


\title{
PACIFIC JOURNAL OF MATHEMATICS
}

\author{
EDITORS
}

\author{
Robert Osserman \\ Stanford University \\ Stanford, California \\ M. G. Arsove \\ University of Washington \\ Seattle 5, Washington
}

\author{
J. Dugundji \\ University of Southern California \\ Los Angeles 7, California \\ Lowell J. Paige \\ University of California \\ Los Angeles 24, California
}

\section{ASSOCIATE EDITORS}
E. F. BECKENBACH
B. H. NEUMANN
F. WOLF
K. YOSHIDA

\section{SUPPORTING INSTITUTIONS}

\author{
UNIVERSITY OF BRITISH COLUMBIA \\ CALIFORNIA INSTITUTE OF TECHNOLOGY \\ UNIVERSITY OF CALIFORNIA \\ MONTANA STATE UNIVERSITY \\ UNIVERSITY OF NEVADA \\ NEW MEXICO STATE UNIVERSITY \\ OREGON STATE UNIVERSITY \\ UNIVERSITY OF OREGON \\ OSAKA UNIVERSITY \\ UNIVERSITY OF SOUTHERN CALIFORNIA
}

\author{
STANFORD UNIVERSITY \\ UNIVERSITY OF TOKYO \\ UNIVERSITY OF UTAH \\ WASHINGTON STATE UNIVERSITY \\ UNIVERSITY OF WASHINGTON \\ AMERICAN MATHEMATICAL SOCIETY \\ CALIFORNIA RESEARCH CORPORATION \\ SPACE TECHNOLOGY LABORATORIES \\ NAVAL ORDNANCE TEST STATION
}

Mathematical papers intended for publication in the Pacific Journal of Mathematics should by typewritten (double spaced), and on submission, must be accompanied by a separate author's résumé. Manuscripts may be sent to any one of the four editors. All other communications to the editors should be addressed to the managing editor, L. J. Paige at the University of California, Los Angeles 24, California.

50 reprints per author of each article are furnished free of charge; additional copies may be obtained at cost in multiples of 50 .

The Pacific Journal of Mathematics is published quarterly, in March, June, September, and December. Effective with Volume 13 the price per volume (4 numbers) is $\$ 18.00$; single issues, $\$ 5.00$. Special price for current issues to individual faculty members of supporting institutions and to individual members of the American Mathematical Society: $\$ 8.00$ per volume; single issues $\$ 2.50$. Back numbers are available.

Subscriptions, orders for back numbers, and changes of address should be sent to Pacific Journal of Mathematics, 103 Highland Boulevard, Berkeley 8, California.

Printed at Kokusai Bunken Insatsusha (International Academic Printing Co., Ltd.), No. 6, 2-chome, Fujimi-cho, Chiyoda-ku, Tokyo, Japan.

PUBLISHED BY PACIFIC JOURNAL OF MATHEMATICS, A NON-PROFIT CORPORATION

The Supporting Institutions listed above contribute to the cost of publication of this Journal, but they are not owners or publishers and have no responsibility for its content or policies. 


\section{Pacific Journal of Mathematics}

\section{Vol. 14, No. $2 \quad$ June, 1964}

Tom M. (Mike) Apostol and Herbert S. Zuckerman, On the functional equation $F(m n) F((m, n))=F(m) F(n) f((m, n)) \ldots \ldots \ldots \ldots \ldots \ldots \ldots \ldots \ldots \ldots \ldots$

Reinhold Baer, Irreducible groups of automorphisms of abelian groups . . . . . . . 385

Herbert Stanley Bear, Jr., An abstract potential theory with continuous kernel . . . . 407

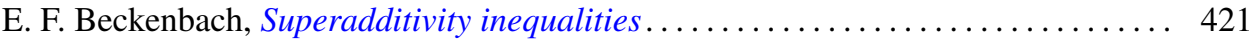

R. H. Bing, The simple connectivity of the sum of two disks . . . . . . . . . . . 439

Herbert Busemann, Length-preserving maps ...................... 457

Heron S. Collins, Characterizations of convolution semigroups of measures . . . . . . 479

Paul F. Conrad, The relationship between the radical of a lattice-ordered group and complete distributivity............................ 493

P. H. Doyle, III, A sufficient condition that an arc in $S^{n}$ be cellular . . . . . . . . . 501

Carl Clifton Faith and Yuzo Utumi, Intrinsic extensions of rings . . . . . . . . . . 505

Watson Bryan Fulks, An approximate Gauss mean value theorem . . . . . . . . . . 513

Arshag Berge Hajian, Strongly recurrent transformations . . . . . . . . . . . . . 517

Morisuke Hasumi and T. P. Srinivasan, Doubly invariant subspaces. II . . . . . . . 525

Lowell A. Hinrichs, Ivan Niven and Charles L. Vanden Eynden, Fields defined by

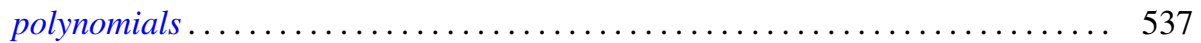

Walter Ball Laffer, I and Henry B. Mann, Decomposition of sets of group

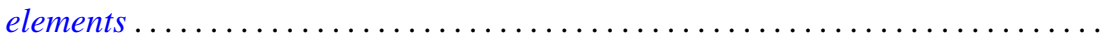

John Albert Lindberg, Jr., Algebraic extensions of commutative Banach

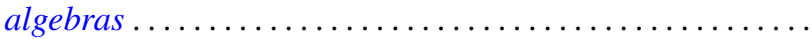

W. Ljunggren, On the Diophantine equation $C x^{2}+D=y^{n} \ldots$

M. Donald MacLaren, Atomic orthocomplemented lattices ....

Moshe Marcus, Transformations of domains in the plane and applications in the

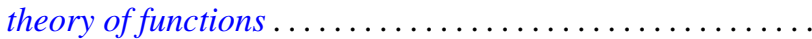

Philip Miles, $B^{*}$ algebra unit ball extremal points . .................. 627

W. F. Newns, On the difference and sum of a basic set of polynomials . . . . . . . 639

Barbara Osofsky, Rings all of whose finitely generated modules are injective ...... 645

Calvin R. Putnam, Toeplitz, matrices and invertibility of Hankel matrices . . . . . . . 651

Shoichiro Sakai, Weakly compact operators on operator algebras . . . . . . . . . 659

James E. Simpson, Nilpotency and spectral operators . . . . . . . . . . . . . 665

Walter Laws Smith, On the elementary renewal theorem for non-identically

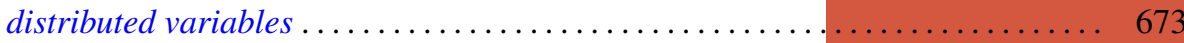

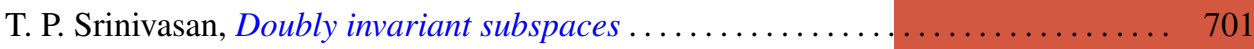

J. Roger Teller, On the extensions of lattice-ordered groups . . . . . . . . . . . . 709

Robert Charles Thompson, Unimodular group matrices with rational integers as

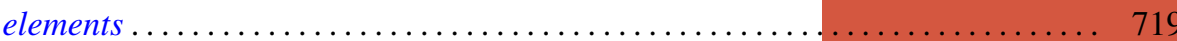

J. L. Walsh and Ambikeshwar Sharma, Least squares and interpolation in roots of unity

Charles Edward Watts, A Jordan-Hölder theorem .................... 731

Kung-Wei Yang, On some finite groups and their cohomology .............. 735

Adil Mohamed Yaqub, On the ring-logic character of certain rings ............ 741

Paul Ruel Young, A note on pseudo-creative sets and cylinders 